\title{
SOBRE UM CASO DE SYNDROMA INFUNDIBULO-TUBERIANO LUETICO
}

\author{
SYLVIO BERTACCHI
}

A observação que vou relatar é de uma doente observada no Posto de Syphilis Arnaldo Vieira de Carvalho.

Tratando-se de cibente de ambulatorio, não foi observada diariamente e algumas provas deixaram de ser feitas por esse motivo.

A doente nos appareceu no dia 3 de setembro de 1933, vinda da 1. ${ }^{a}$ Medicina de Mulheres onde estivera internada e trazia como unica indicação um Wassermann com $(++++)$.

A sua observação é a seguinte: sada.

C. R. G., com 29 annos de idade, domestica, portugueza, ca-

QUEIXA: Dores de cabeça intensas, sêde tambem intensa, poliuria, amenorrhéa e perturbações visuaes.

HISTORIA PREGRESSA DA MOLESTIA ACTUAL: Não precisa bem a data de inicio da molestia, sabe que foi nos fins de 1932.

Após uma grippe, com dores lombares e febre, tratada por medico, começou a sentir grande sêde, que se intensificava dia a dia, a ponto de se tornar martyrizante quandio não satisfazia a sua vontade de beber. Em pouco tempo chegou a beber só durante a noite 6 garrafas de agua, durante o dia bebia mais do que isso, chegando a beber ao todo nas 24 horas, 12 litros de agua.

Começou tambem a urinar com muita frequencia; ultimamentc quando a doença era mais forte, de $15 \mathrm{em} 15$ minutos, mesmo durante a noite. A urina era clara como agua.

Pouco tempo depois do inicio da molestia tornou-se amenorrheica, não sabendo precizar quanto tempo depois; na mesma occasião começou a ter fortes dores de cabeça, sob fórma de pontadas fortissimas bem no alto da cabeça, irradiando-se para o lado direito, e outras vezes para traz, descendo pela espinha até a 2.* 
ou 3." vertebras dorsaes. Passada a pontada permanecia uma dôr forte durante uns 10 minutos que ia se attenuando em seguida.

Ficava depois com a cabeça tonta, dolorida e pesada, sentia necessidade de deitar-se

Essa pontada tinha-a diversas vezes no dia e mesmo durante a noite; se estava deitada parecia que era mais forte, levantava-se $\epsilon$ andava de um lado para outro, tonta e cambaleante.

A dòr de cabeça que restava augmentava muito com a tosse c com os espirros.

$\mathrm{Na}$ primeira vez que teve as pontadas referidas, perdeu a visão completamente; esta voltou um pouco depois da crise, porém nunca mais enxergou bem, ficou com a visão tão diminuida a ponto de distinguir só os vultos das pessoas da casa e de apenas reconhecel-as pela voz.

Andava pela casa, muito fraca, com muita sêdie, urinando muito, mas cada vez que lhe dava a pontada na cabeça, o que se fazia algumas vezes no dia como já verificamos, ficava completamente céga e era conduzida pela mão por pessoa da casa. A vista nunca voltou completamente, melhorava um pouco para logo perdel-a com a pontada successiva.

Depois do inicio das dores referidas, não sabe quando, sobreveiu-lhe tambem grande somno, era irresistivel, dava-lhe algumas vezes no dia, dormia mesmo sentada e de uma vez no bonde quando a levavam ao medico.

Néga febre, um medico que a mediu no inicio da molestia achou-a com 37 gráus.

Durante quase um anno de doença consultou diversos medicos, todos lhe deram injecções e remedios por bocca sem que com elles obtivesse melhoras.

Entre outros medicamentos tomou Optarson e Thiobi.

Um exame de urina feito a pedido de um clinico, em 26 de dezembro de 1932, no inicio da molestia, portanto, revelou: Densidade 1002,7, $\mathrm{NaC1}$, por litro, 1,46 gr. Traços de albumina. Ausencia de glicóse. Sedimento: corpusculos de pús e algumas he-
matias.

No dia 15 de setembro ingressou na 1.a Medicina de Mulheres. Nessa enfermaria fizeram-lhe Wassermann no sangue e o resultado foi $(++\dot{+}+)$. A ciensidade da urina era de menos de 1001 . Deram-lhe duas injecções de "914", com as quaes melhorou bastante em todos os symptomas.

Teve alta, a pedido, em 29 de setembro, sendo encaminhada ao Posto de Syphilis para continuar o tratamento.

ANTECEDENTES PESSOAES : E empregada em serviços domesticos, sempre teve saúde, não é ethylista, bebe pouco café. 
Pesava antes da molestia 56 kilos, hoje pesa 52 k. e 500 grs.

Néga molestias venereo-syphiliticas. Néga outras molestias inffectuosas a não ser sarampo quando criança e grippe algumas vezes.

E menstruada desde os 13 annos, com regularidade, durando o catamenio 4 a 5 dias.

ANTECEDENTES HEREDITARIOS: Pae fallecido ha 14 annos de desastre; tinha ficado nos ultimos annos de sua vida, meio bôbo e meio esquecido. Pela descripção que nos faz a paciente, da molestia do pae, pensamos tratar-se de um caso de $\mathrm{Pa}$ ralyzia Geral.

Tem 6 irmãos; uma irmã, a mais velha, soffreu de ataques epylepticos durante alguns annos, até que ha 14 annos fizeramlhe tratamento anti-syphilitico; nunca mais teve ataques.

Todas as irmãs em numero de 4 , a cada instante tem abortos, e os filhos que conseguem ter são fracos.-(sic)

Mãe viva, com mais de 70 annos, é relativamente forte.

A paciente é casada, separada porém do marido ha 3 annos, não sente necessidade de relações sexuaes. Teve um filho que morreu aos 3 mezes de idade, com meningite. Néga abortos.

INTERROGATORIO SOBRE OS DIFFERENTES APPARELHOS: CABEÇA - soffria de cephaleias uma vez ou outra. OUVIDOS e NARIZ: nada conta para o lado desses apparelhos. OLHOS: o primeiro mal foi o que sobreveiu no decurso da molestia actual. GARGANTA : nada.

CARDIO RESPIRATORIO: conta tosse algumas vezes, curadlas expontaneamente.

GASTRO INTESTINAL: sempre teve bom appetite, bôa digestão. Depois do inicio da molestia actual, perdeu em parte o appetite e tem ligeira prisão de ventre. Néga vomitos ou qualquer outro disturbio.

GENITO URINARIO: Antes da molestia actual nunca teve qualquer perturbação da menstruação. Néga perturbação para o lado desse apparelho, antes da actual molestia.

ASPECTO GERAL: A doente apresenta ligeiro estado de apathia, a physionomia é de soffredora e percebe-se que tem perturbações visuaes, porque fixa muito o olhar como quem procura enxergar.

A paciente é de typo mediolineo, de desenvolvimento physico normal, com bôa constituição osséa e muscular. A marcha da paciente é prejudicada pela diminuição da visão, assemelha-se um pouco a marcha ébriosa batendo frequentemente nas paredes. Não passa uma porta sem nella esbarrar. 
SYSTEMA GANGLIONAR : não ha ganglios epitrochleanos nem cervicaes. Não ha esternalgia. Ha ligeira tibialgia.

TEMPERATURA : foi verificada algum tempo após ter sido feita esta observação e apresentou differença de 0,6 decimos entre as duas axillas.

Temp. na axilla esquerda 36,8 gráus.

Temp. na axylla direita 36,2 gráus.

A temperatura tirada noutras occasiões, revelou sempre a mesma differença de mais ou menos 0,6 decimos.

EXAME ESPECIAL: CRANEO, é symetrico, não tem pontos dolorosos. OLHOS: não ha anisocoria; as pupillas reagem a luz sendo que a direita o faz vagarosamente. Reflexos á distancia e á accommodação, são normaes. Musculatura extrinseca integra. ORELHAS, NARIZ, SEIOS DA FACE: nada apresentam. BOCCA, mucósa de colloração normal ; lingua sem saburro, dentes regularmente conservados.

PESCOÇO : nada apresenta, THYREOIDE, de consistencia e tamanho normaes.

THORAX: symetrico, nada revela pelo exame dos pulmões.

CORAÇAO: Nota-se pela ausculta, um tymbre metallico intenso da segunda bulha no fóco aortico.

ARTERIAS: de consistencia normal. Pulso radial rythmico e isochrono. Pressão arterial medidia pelo TYCOS, Mx. 100 Mn. 62.

ABDOMEN : nada revela.

FIGADO: não é palpavel abaixo do rebordo.

BAÇO: não é palpavel.

COLONS: palpaveis, sem nada de particular. O cecum é ligeiramente doloroso á palpação.

EXAME GYNECOLOGICO: (feito por especialista) Orgãos genitaes externos normaes. Vagina: ampla para dois dedos. Utero: A. V. F. movel. Annexos: livres. Hysterometria: $7 \mathrm{cms}$.

SYSTEMA NERVOSO - estatica e equilibrio : não ha signal de Romberg. A marcha é ligeiramente ebriosa, como já descrevemos.

TONUS MUSCULAR: conservado. Não ha rigidez da nuca.

FORÇA MUSCULAR: um tanto diminuida, facto este verificavel e revelado pela paciente.

TREMORES: não ha.

SENSIBILIDADE : á dôr, calor e pressão; normaes. A doente localiza bem as impressões. Sensibilidade da cornea, perfeita. Segmentar, idem. Não ha dismetria. 
REFLEXOS: Pupillas, (já descriptos no exame de olhos).

Patelares: vivissimos, zona reflexogena augmentada, principalmènte do lado esquerdo, onde é obtido em quase toda a crista cia tibia.

Aquilianos: vivos. Signal de Babinsky, ausente.

Refflexos bicipitaes, tricipitaes, estylo radiaes e cubito pronadores, muito vivos cilos dois lados.

Não ha clonus da rotula, nem do pé.

Reflexos cutaneos abdominaes: presentes.

\section{Exames complementares}

Estes exames foram feitos quando o tratamento já iniciado e a symptomatologia diminuida.

URINA : clara, assemelhando-se a agua, tendo apenas uma tonalidade amarellada. Densidade, 1002. Albumina, traços leves. Assucar, não contem. Chloretos, 2,10 o|oo Ureia, 8 grs. o|oo. Sedimento: cellulas epyteliaes, traços de pús, urato de sodio.

REAÇAO DE WASSERMANN no sangue, fortemente positiva $(++++)$.

LIQUIDO CEPHALO RACHIDIANO: Dr. O. Lange, (12 de setembro, 1933):

Punção sub-occipital, deitada.

Pr. inicial, 6. Pr. final, 0.

Liquido limpido, incollor. Volume, 18 c. c.

Cytologia, 3,2.

Albumina, 0,30 .

Chloretos, 6,90.

Pandy, Nonne, Weichbrodt, negativos.

Benjoin colloidal 0000000000000000 .

Takata-Ara, negativo.

Wassermann, negativo com 1 c. c.

RADIOGRAPHIA DA SELlA TURCICA: Dr. Paulo de Toledo, (12 de setembro, 1933).

Sella turcica de contornos, profundidade, abertura, normaes.

Clinoides anteriores e posteriores, conservadas. Pontos das pyramides de aspecto normaes. Sulcos vasculares de apparencia normal. Ligeiro augmento das impressões digitaes e ligeiro affastamento da sutura lambdoide.

DIAGNOSTICO: Signaes radiologicos de pequeno augmento da pressão endo-craneana.

EXAME OPHTAlmológico: Dr. Durval Prado, 7 de setembro, 1933). (fig. 1).

Campo visual: Hemianopsia homonyma direita incompleta. 
$O$ exame do fundo de olho e da papilla optica foi normal. DOSAGEM DE CHLORO E CHLORETOS: no sangue. Dr. Cerrutti.

Chloro, 3,55 oloo.

Chloretos, 5,85 ojoo, dentro dos limites normaes, portanto.

Em resumo, a nossa doente apresentava:

a) Polydipsia.

b) Polyuria com hypostenuria e hypocloruria.

c) Amenorreia.

d) Cephaleas intensas.

e) Hemianopsia homonyma direita.

f) Narcolepsia.

Phenomenos estes dispostos na ordem chronologica de apparecimento. Além disso, pelo exame constatámos reflexos patellares vivos com augmento da zona reflexogena, sem signal de $\mathrm{Ba}$ binsky e uma differença thermica entre as duas axillas.

Esta symptomatologia é tão numerosa que sem que procuremos estabellecer um diagnostico differencial, ella nos conduz aos centros lesados. De facto, se considerassemos isoladamente os symptomas apresentados, poderiamos pensar numa polyuria nervosa ou hysterica, numa amenorreha gravidica ou por insufficiencia ovariana; poderiamos attribuir perturbações occulares numa mulher amenorreica á gravidez, mas no nosso caso, além dos exames negativos feitos por especialista, para os outros symptomas afresentados não haveria explicação plausivel.

Os centros vegetativos, reguladores do equilibrio hydrico, do somno, o thermo-regulador, metabolicos, da menstruação, estão tâo proximos entre si, assim como as vias opticas, que um exame apenas desses symptomas nos conduz para a região Infundibulotuberiana. Autores ha que descreveram nessa região 32 centros regetativos.

Lhermitte, que muito bem estudou essas symdromas, diz: Quando num adulto se agrupam os seguintes symptomas de ordem vegetativa, como nos doentes que estudou e achou grupados; polyuria, polydipsia, boulemia, desordens sexuaes, desordens da funcção hypnica, e mais particularmente hypersomnia com cataplexia, obesidade, amenorreia ou impotencia; o diagnostico topographico da lesão está, por assim dizer, inscripto na physionomia do doente e hoje nem um observador advertido hesitará em situar a alteração na região ventral do diencephalo. 
Além disso, a via optica é um dos systhemas mais lesados, attingida seja ao nivel do chiasma, seja ao nivel das bandielletas opticas.

Algumas vezes, mas raramente observamos perturbações motoras, hemiparesia ou paraparesia com exaggero dos reflexos e signal de Babinsky, ligado a alteraçốes das fibras cortico-espinaes ao nivel do pé peduncular.

A symdroma acima; polyuria, polydipsia, boulemia, desordens hypnicas, amenorrheia ou impotencia segundo se trate de mulher ou de homem, constitue para Lhermitte a symdroma Infundibulotuberiana global. Descreve elle porém, symdromas Infundibulotuberianos dissociados em que existe o diabete insipido (o mais frequente na symthomatologia) associado quer á adiposidade quer a hypersomnia.

A ordem e o tempo de apparecimento desses symtomas não é sempre a mesma, como se pódle verificar nas publicações sobre o assumpto; $\mathrm{e}$ isto é natural pois que o processo quando iniciado, póde se expandir ora para um lado ora para outro, attingindo este ou aquelle centro do tuber cinereum ou do infundibulo ou limitar-se a pontos determinados.

Sobre esse particular esse autor descreve o caso de uma mulher com diabete insipido e emmagrecimento e que só depois, annos mais tarde, apparece adiposidade e perturbações hypnicas.

Weissembach, 'Gilbert-Dreyfus, apresentam um caso de uma moça com 20 annos que em 1915 teve menopausa brusca e definitiva, paralysia do VI par craneano, hemiplegia, polyuria de 10 a 14 litros nas 24 horas. A reaç̧ão de Wassermann foi positiva. Tratou-a pelo "914" desapparecendo só a hemiplegia. Em 1923, 8 annos mais tarde, portanto, sobreveiu keratite intersticial e adiposidade, augmentando a paciente 15 kilos. Em 1931, rarefação dos pellos e atrophia do utero, perturbação glyco-reguladora e thermo-reguladora, isto é: uma febre inversa, $38^{\circ}$ pela manhã e $37^{\circ}$ á noite.

Esses dois casos vem attestar o que acima dissemos, pois a ordem de apparecimento dos symptomas não foi sempre a mesma e se succederam em intervallos bem grandes.

Assim senço se a nossa doente não fôr persistente no tratamento e o processo vier a progredir, poderá vir a ter um dia, adiposidade genital, boulemia, etc.

\section{Etiologia e Discussão}

As symdromas Infundibulo-tuberianas podem ser produzidas por:

a) Tumores; primitivos ou metastaticos.

b) Traumatismos. 
c) Tuberculose da base.

d) Encephalite lethargica.

e) Syphilis; adquirida ou hereditaria.

No nosso caso, podemos excluir os traumatismos, a tuberculose e a encephalite, quer pelos commemorativos da doente, quer pelos exames levados a effeito, mas principalmnte pelos commeinorativos.

Resta-nos differenciar entre tumor e syphilis.

Couturat, diz que os symptomas que fallam em favor de lesão tumoral são sobretudo signaes de hypertensão intra-craneana, taes como vomitos cerebraes, crises comiciaes, cephaleas, vertigens, bradycardias. O tumor determina signaes occulares frequentes: amblyopia progressiva, evoluindo para a cecidade absoluta por attingir os nervos opticos, symdromas de hemianopsia bi-temporal por attingir o chiasma, ou hemianopsia homonyma bi-lateral por attingir a bandelleta optica. A radiographia vem affirmar o diagnostico de tumor mostrando alargamento da sella turca, gasto da lamina quadrilatera e das apophyses clinoides posteriores.

Lhermitte, differencia nessas symdromas a syphilis da neoplasia basilar, assim: As cephaleias são raramente observadas ou não attingem a intensidade que se verificam no curso de neoplasias. Geralmente não ha estase papilar na syphilis; falta ainda hypertensão intra-craneana e a punção não mostra hypertensão comparavel a que se vê nas neoplasias. Quanto aos signaes radiographicos, diz a mesma coisa que já verificamos em Couturat, isto $\dot{e}$, que os tumores affectam a sella turca ou as clinoides, emquanto que na syphilis o aspecto dessa região é normal.

Weissembach ainda é concord'e com os autores acima, dizendo que a symdroma Infunclibulo-tuberiana de etiologia syphilitica se differencia do de origem tumoral porque este produz hypertensão intra-craneana e hemianopsia por compressão chiasmatica, a menos que não existam formações gomosas de volume importante.

No nosso caso teriamos a favor de tumor as cephaleas intensas. Quanto á pressão do liquor; foi verificada por punção já no inicio do tratamento e quando as dôres de cabeça já não existiam, revelando-se normal. (Pi, 6. P. f., O. Punção, S. O. D.)

A radiographia revelou ligeira hypertensão craneana pelas impressões digitaes; ora, como essas impressões demoram a desapparecer, é possivel que ellas ainda existissem no dia em que foi feita a radiographia, apezar do desapparecimento da causa que a motivou por effeito do tratamento.

Parece-nos que as cephaleas como apresentava a nossa paciente, em crises, irradiando-se para a columna e augmentando 
com a tosse e com os espirros, possam ser attribuidos á hypertensão do liquido cephalo rachidiano, não se podlendio porém excluir causas outras de cephaleas, porque, essa hypertensão era ligeira como assignalou o radiologista; não havia estase papilar, consequencia frequente das grandes hypertensões tumoraes; não havia vomitos cerebraes na historia da paciente, facto que acompanha tambem frcquentemente as cephaleas hypertensivas.

Se por um lado a radiographia nos lembra a hypothese de tumor, por outro nos dá signaes contrarios a processo dessa nature$\mathrm{za}$, pois demonstrou integras as apophises clinoides e a lamina quadrilatera, e de tamanho normal a sella turca.

O liquidio cephalo rachidiano demonstrou negativas as reações para syphilis, porém não firmou a hypothese de tumor; apresentou apenas um ligeiro augmento das cellulas, 3,2 por $\mathrm{mm}^{3}$., e um ligeiro augmento das albuminas, 0,30 o|oo.

Não ha coisa alguma que nos sugira a hypothese de tumor; nem uma dissociação albumino-cytologica, nem uma modificação no aspecto do liquido que se torna frequentemente xantochromico.

Não existe tambem signal de syphilis, a não ser o pequeno augmento da albumina e da cytologia; as reacções especificas foram negativas.

Os processos tumoraes ou syphiliticos do cerebro, podem porém evoluir sem ou com pequenas módificações liquoricas, donde o resultado da punção no nosso caso não affirmar nem inffirmar qualquer um dos dois processos.

Os signaes de syphilis encontrados em nossa paciente; clangor da $2 .^{a}$ bulha aortica, tibialgia, antecedentes familiares syphiliticos, e reacção de Wassermann no sangue fortemente positiva, fallam a favor dessa etiologia. Resta-nos a prova mais importante a favor de um processo syphilitico; a therapeutica especifica deu optimo resultado.

Quanto ao exaggero dos refflexos patellares, e augmento das zonas reflexogenas observadas na nossa paciente, sem signal ie Babinsky, póde ser devido como já lembrando Lhermitte em capitulo anterior dissemos, a alterações das fibras cortico-espinaes ao nivel do pé do pedunculo.

A hemianopsia homonyma direita, é diada por um provavel comprometimento das fibras da bandelleta optica esquerda; provavel digo, porque só a prova de Wernicke ou da rigiclez pupillar hemianoptica, poderiam esclarecer com a ausencia ou presença do reflexo pupilar á luz, se a lesão é da bandelleta optica ou dos centros opticos do lobo occipital. 
Essa prova deixou de ser feita não só porque a technica é muito difficil, mas tambem porque sendo a hemianopsia incompleta, os resultados seriam duvidosos.

Como já concluimos acima por uma lesão na base do $3 .^{\circ}$ ventriculo é mais provavel tambem que a lesão optica esteja situada na bandelleta e não nas radiações opticas ou nos centros opticos do lobo occipital, porque para tanto precisaria que existisse mais de uma lesão, ou então que esta fosse muito extensa.

Estabelecido o diagnostico de symdroma Infundibulo-tuberiano de etiologia luetica, resta-nos estabelecer o provavel processo patologico. Trata-se de uma gomma, de uma meningite da base, ou de uma vascularite?

Lhermitte e Barbonneix, Lhermitte e Kaplan, em diversos casos autopsiados, encontraram sempre meningo-vascularites syphiliticas da base, lesando o assoalho do $3 .^{\circ}$ ventriculo e os nucleos tuberianos, com integridadie da hypophise.

Couturat, tambem encontrou mais frequentemente lesões meningo-encephalicas, raramente se tratava de gommas ou de osteoperiostite da base.

No caso em apreço, não encontramos pelos Raios X, signal algum de osteo-periostite ou de gomma compromettendo o tecido osseo da base, o que não exclue absolutamente a hypothese porque uma gomma localizada nos tecidos moles não daria signaes radiologicos.

A maior frequencia das vascularites e a ausencia de reacções liquoricas que denunciariam uma provavel meningite, nos inclina a pensar num processo daquella natureza, apezar do comprometímento da bandelleta optica esquerda e dos signaes radiographicos de hypertensão, que como já estudamos são proprios dos tumores e das gommas.

\section{Evolução e tratamento}

Qualquer dos especificos da syphilis, póde ser usado na symdrome Infundibulo-tuberiano. A nossa doente já tinha iniciado tratamento salvarsanico, nós entretanto, com mais prudencia continuamos o tratamento pelo Cyaneto de Mercurio, e o resultado foi bom. a $1 / 2 \%$.

Foi começada uma série de dois c. c. de Cyaneto de Mercurio

Dia 17 de setembro de 1933 - a doente melhora dia a dia, já tomou 7 injecçōes de Cyaneto, a sêde e a polyuria diminuiram consideravelmente, não tem mais crises de narcolepsia, está sa- 
tisfeita, a vista volta-lhe aos poucos, já consegue enfiar linha na agulha, coisa que não fazia desdè o inicio da molestia e já consegue coser.

Urina desse dia, 4 litros e $\mathrm{x} / 2,-$ Densidade 1005, - Chloreto de Sodio 2,43 oloo, - Ureia 13,62 oloo.

Como se póde verificar, a diurese baixou bastante.

Dia 24 de setembro de 1933 - tendo já a doente completado a sua 10. ${ }^{a}$ injecção de Cyaneto, julgamos conveniente iniciar o tratamento pelo "914". A primeira dose foi feita a 24 de setembro e foi de 0,15 ctgr. Fez a partir dessa data semanalmente uma injeç̧ão, de dloses crescentes até attingir 0,60 ctgr. por vez.

Dia 1 de outubro de 1933 - Urina: quantidade, 3 litros e $1 / 2$ - Densidade, 1003 - Albumina, traços levissimos - Chloretos em NaC1. 4,44 o|oo.

Dia 20 de outubro de 1933 - Novo exame do campo visual revelou-o mais amplo, como se póde verificar na Fig. 2.

Dia 5 de novembro de 1933 - A doente está muito contente. sente melhoras diarias. Arranjou emprego no principio do mez e recomeçou a trabalhar. Durante a noite só se levanta duas vezes no maximo para urinar. Em exame do systhema nervoso, encontramos todos os reflexos no mesmo estado. A pupilla direita já reage normalmente á luz.

Dia 26 de novembro de $19^{3} 3$ - Exame de urina: quantidade 3 litros e 200 c. c. - Densidade, 1004 - Chloretos em NaC1. 3.51 oloo - Albumina, traços minimos. Continua, pois, a diurese a diminuir assim com a sêde. A unica coisa de que se queixa ainda a nossa doente, é da Amenorreia.

Dia 3 de dezembro de 1933 - Nesta data a doente já havia feito 5,55 gr. de "914", a sua veia tornou-se difficil de apanhar. Suspendemos o tratamento salvarsanico, pretendendo continual-o pelo Bismuto. Antes, porém, pedimos á doente que fizesse novos exames.

Dia 6 de dezembro de 1933 - Novo exame do campo visual, ainda feito pelo Dr. Durval Prado, revela melhoria, como se póde verificar na Fig. n. 3.

Dia 17 de dezembro de 1933 - Exame de urina feito pelo Dr. H. Cerrutti. Quantidade 3 litros, - Côr, amarella citrina, Aspecto, ligeiramente turva, - Cheiro, "sui-generis", - Sedimento, minimo. Densidade, 1005. - Reacção ao Tornesol, amphotera. - Albumina, traços minimos. - Assucar, não contem. Acetona, não contem. - Sedimento : cellulas epiteliaes chatas, cy- 
lindroides. leucocitos (traços de púz). Chloreto cile Sodio, 1,87 lindroides. leucocitos (traços de pún - Phosphatos em $\mathrm{P}^{2} \mathrm{O}^{5} ; 0,25$
gr. oloo. - Ureia, 8,0 gr. oloo. -
gr. oloo.

A doente sente-se curada. Apezar da nossa recommendaçâo de ser constante no tratamento, abandona-o sob pretexto de o serviço nāo o permittir.

Dia 1 de fevereiro de 1934 - Fazem dois mezes que tomou a ultima injecção de "914". Volta, dizendo que a sêde augmentou novamente, bebendo 6 garrafas de agua nas 24 horas. Conseguimos por gentilleza do Dr. Samuel Ribeiro, o Metabolismo Basal a 2-2-934. Esse exame foi feito em bôas condições e revelou:

a 2-2-934. Esse exabolismo Basal, - 1,1 \%, dentro dos limites normaes, portanto.
Dia 4 de fevereiro de 1934 - Urina, quantidade, 5 litros. Dia 4 de fevidade, 1003 . - Chloretos, 1,87 oloo. - Sedimento, cellulas
epiteliaes chatas, cylindroides, leucocitos degenerados, crystaes de Oxalato de Calcio.

O exame dos refflexos por essa occasião mostrou-os todos vivos, as pupillas reagindo bem a luz e a accommodação. $O$ peso da paciente permanece o mesmo, tem trabalhado sempre e o que a fez voltar foi a nova exacerbação da polydipsia e polyuria.

Receitamos injecções de Bismophanol (10), que tinhamos como amostras.

Após um mez de descanço demos á doente uma caixa de Iodobisman, para tomar duas injecções por semana, e no intervallo uma de Iodeto de Sodio a $10 \%, 10$ c. c., endovenosas.

Com esse tratamento passa melhor ainda a paciente, a diurese cahiu novamente para 3 litros diarios. Sente-se bôa, preoccupando-lhe apenas a amenorrea.

Dia 13 de abril de 1934 - Revela novas melhoras num novo exame do campo visual. (Fig. n. ${ }^{\circ}$ ).

A doente continua em tratamento, está trabalhando normalmente e prometteu não mais abandonar o tratamento. Abandonou-o, porém. novamente, sob o mesmo pretexto; conseguimos, forém, em meiados de setembro, que fizesse novo Wassermann que revelou-se fracamente positivo $(+)$, negou-se, porém, a fazer nova punção rachidiana. $O$ seu estado de saude continua identico ao que constatámos no ultimo exame (13 de abril)

\section{Prognostico}

O prognostico nesses casos é funcção da precocidade do tratamento; quando feito no inicio podem regredir inteiramente todos os males. O que mais custa a se normalizar são os catamenios. O diabete insipido com frequencia não regride completamente. 


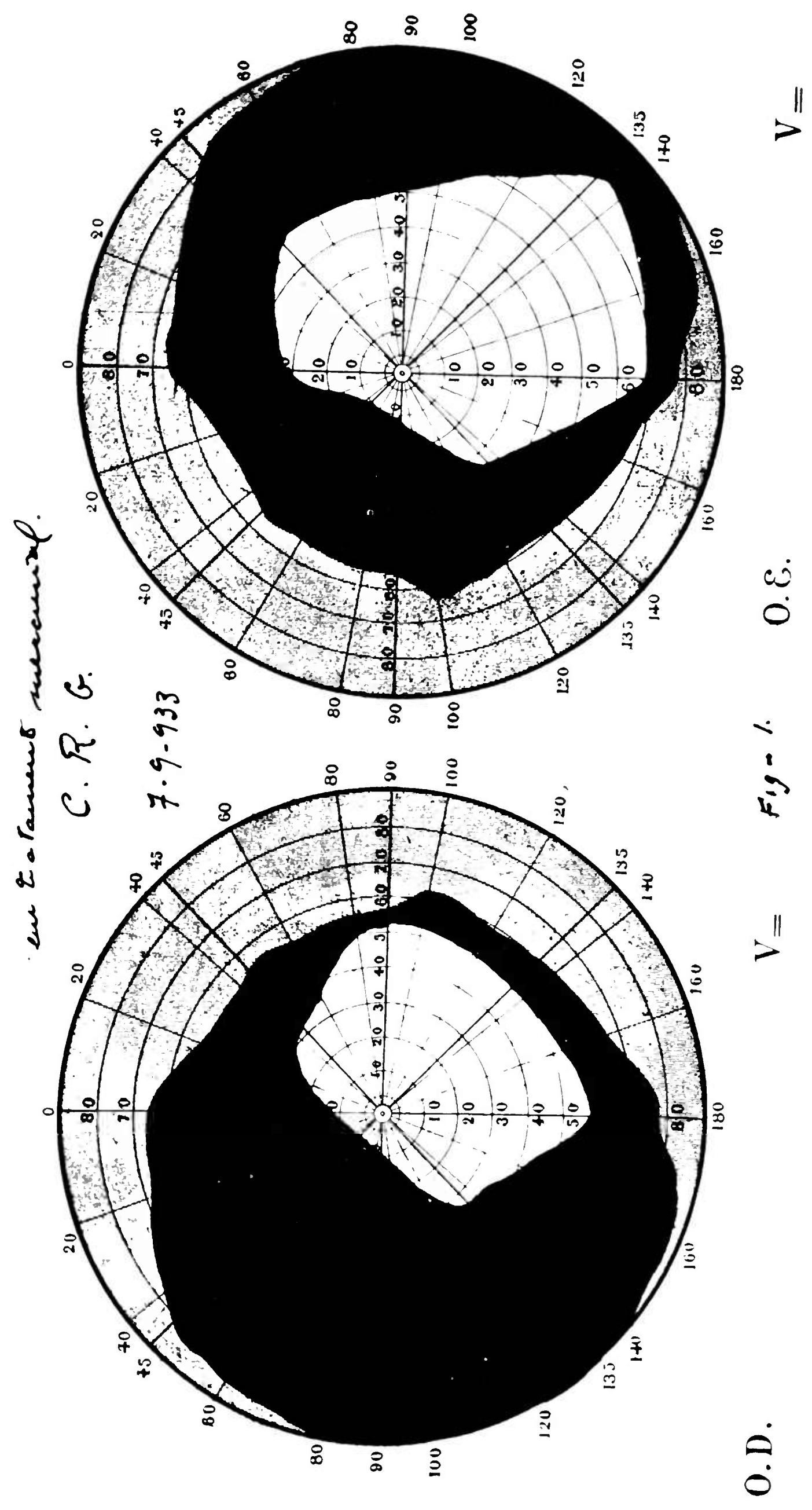





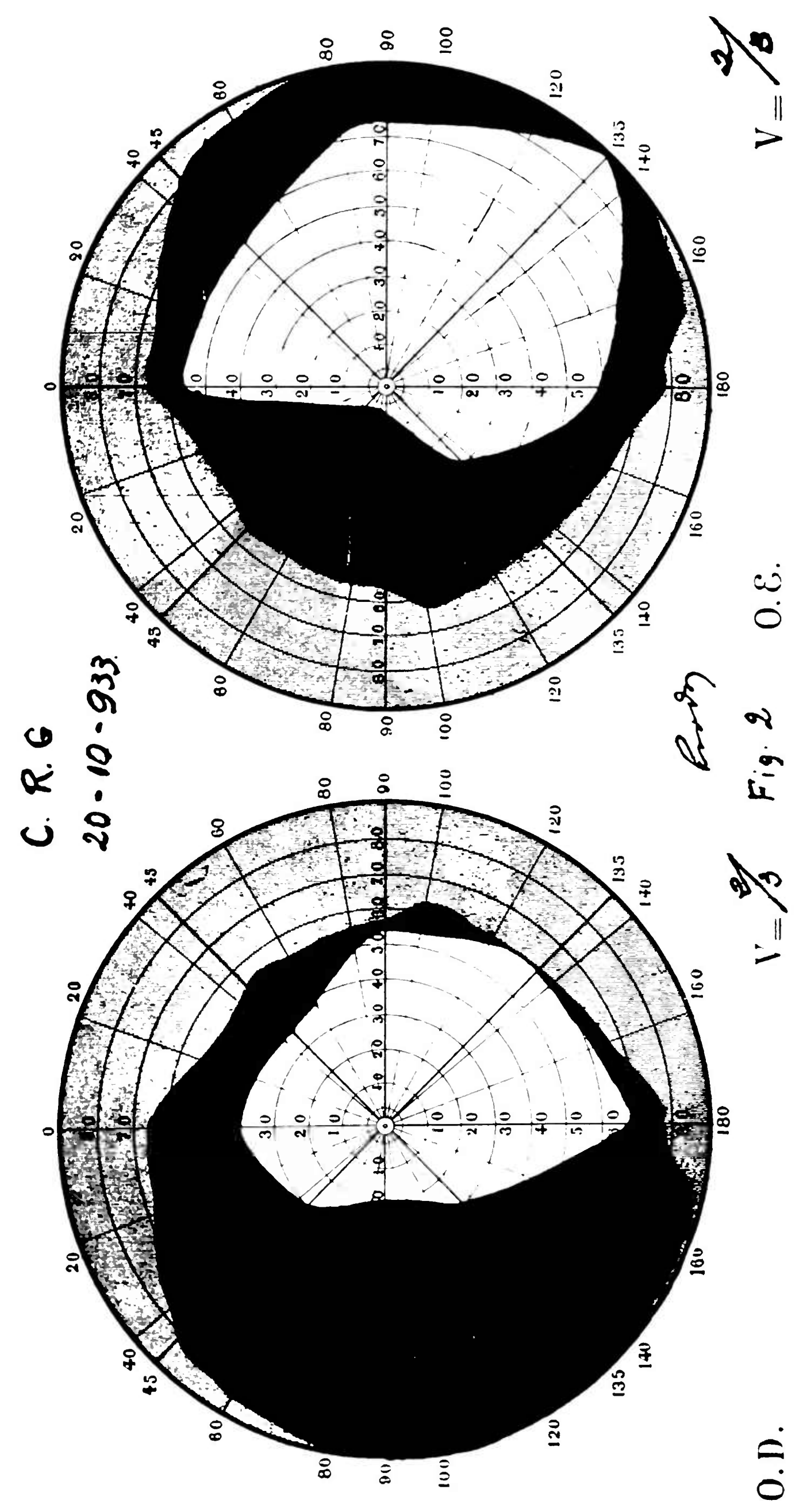





$$
\frac{0}{0}
$$





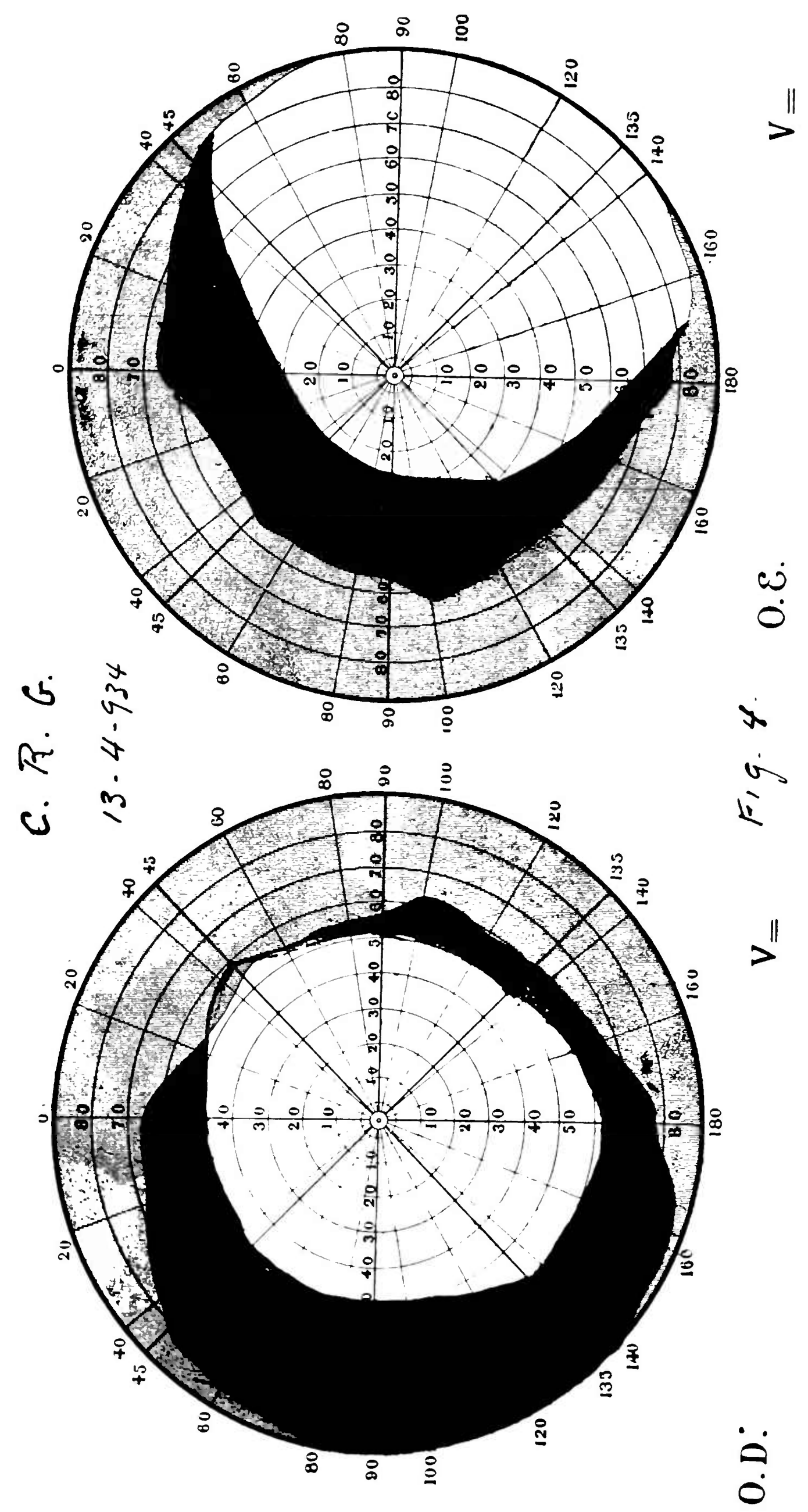





\section{BIBLIOGRAPHIA}

BABONNEIX, L., HUTINEL, J., et AZERARD. Neurosyphilis et diabetis insipidus, Bull. et mem. Soc. Med. d. hôp. de Paris, n. ${ }^{\circ} 14$ March, $9^{3} 3$.

BABONNEIX, L., et LHERMITTE, J., Diabetes insipidrus por encephalite infundibulo-tuberiana de origem syphilitica. C. R. Societé de Biologie n. ${ }^{\circ}$ 36. 1925.

BOCCIA, D., Symdromas neuro-hypophisarios. Rev. sud-am. de endocrinol, n. ${ }^{\circ} 12$, Dec. 1929.

BROUHA, L., Rapports entre l'hypophise et les organes genitaux de la femme, (partie physiologique). Gyneo. et obst. n. ${ }^{\circ}$ 20 Aug. 929.

CASTAIGNE, J., Tumeurs cérébrales. Le journal med., Français. Tom. XX, n. ${ }^{\circ} 10$, Oct. 931.

COUTURAT, J., Le rôle de la syphilis dans la production des syndromes infundibulo-tuberiens. Presse med. 40, 8 june 1932.

CHIARELLO, HEITOR, Considerações clinicas sobre alguns symdromas Infundibulo-hypophisarios. These de São Paulo, 1926.

COTRIM, E. S., Considerações sobre o valor diagnostico das alterações da sella turcica. These de São Paulo, 1930.

jEANSELME et SEZARY, Précis de syphiligraphie et des maladies vénériennes, 1925.

LHERMITTE, J., La syphilis diencéphalique et les syndromes végetatifs qu'elle conditionne; étude clinique. Ann. de med. 33. March, 1933.

LHERMITTE, J., Symptomes des tumeurs du IIIe ventricule et de l'infundibulum. Gaz. d. hôp. 105. 16, nov., 932.

MALAGUETA, IRINEU., Diabetes insipidus. Boletim da Soc. de Med. e Cir. de São Paulo. Semana de conferencias, 1930.

DE MARTEL et GUILLAUME, J., Tumeurs de la region hypophisaire. Rev. neur. n. ${ }^{\circ} 2$, july, 931.

SAHLI, H., Tratado de los métodos de esploracion clinica, 1925. SALMON, A., Quelques donnés experimentales et cliniques á l'appui d'un centre végétatif epileptogéne dans la region diencéphalique. Rev. neur., n. ${ }^{\circ}$, june, 1932. 
SALMOX, A., Le sommeil est-il detérminé par l'excitation d'un centre hipnique ou par la depression fonctionnelle d'un centre de la veille. Rev. neur., n. ${ }^{\circ} 1$, april, 1932.

VAMPRE, E., Estudo clinico das hemyanopsias baseado em 24 observações. Annaes da Fac. de Med., de São Paulo, VI vol., 1931.

YAZ, ROCHA, Endocrinologia, 1933.

WEISSENBACH, R. J. et DREYFUS, G., La syphilis acquise dans le diabete insipide et le syndrome infundibulo-tubérien. Monde med., Paris, 42, may, 1932.

WEISSENBACH: Un cas de syndr. infundibulo-tubérien. Monde med., 15, may, 1932.

WODON, J. L., Etude des rapports entre l'hypophise et les organes genitaux de la femme, (partie clinique). Gynec. et obst., aug., 1929.

ZAGOTTIS, A., Tumores do encephalo e radiotherapia profunda. These de São Paulo, 1931. 